\title{
Physiology of Calcium Nutrition in Plants
}

\author{
Mainu Hazarika ${ }^{1 *}$ and Praveen Kumar $^{2}$ \\ ${ }^{1}$ Department of Horticulture, Assam Agricultural University, Jorhat-785 013, Assam, India \\ ${ }^{2}$ Department of Genetics and Plant Breeding, Lovely Professional University, \\ Phagwara- 144 411, Punjab, India \\ *Corresponding author
}

\section{A B S T R A C T}

\section{Keywords}

Calcium nutrition, $\mathrm{Ca}^{2+}$-ATPases, Apoplastic way

\section{Article Info}

Accepted: 07 September 2020 Available Online: 10 October 2020
Calcium is an essential macro-nutrient in plants, with concentrations in the shoot ranging from 0.1 to $5 \%$ of dry wt. $\mathrm{Ca}^{2+}$ is taken up by the root and transported to the shoot in a mainly apoplastic way to avoid interference with its function as second messenger. To circumvent the casparian strip, it has to enter the cytosol of the endodermal cells via channel proteins and subsequently be exported into the apoplast via $\mathrm{Ca}^{2+}$-ATPases or $\mathrm{Ca}^{2+} / \mathrm{H}^{+}$anti porters. $\mathrm{H}^{+} / \mathrm{Ca}^{2+}$ anti porters and $\mathrm{Ca}^{2+}$-ATPases, which actively deliver $\mathrm{Ca}^{2+}$ into the apoplast or intracellular stores. These channels maintain a low $\left(\mathrm{Ca}^{2+}\right)$ cyt in the resting (un-stimulated) cell appropriate for Cytoplasmic metabolism,restore $\left(\mathrm{Ca}^{2+}\right)$ cyt to resting levels following a $\left(\mathrm{Ca}^{2+}\right)$ cyt perturbation (stimulated). $\mathrm{Ca}^{2+}$ contributes to the structure of cell wall, stability of cell membrane, photosynthetic reaction, photo-protection (D1 protein, PsbS regulated by $\mathrm{Ca}^{2+}$ ), stomatal movement and pollen tube growth and elongation. Calcium sensors, including calmodulin (CaM), calmodulin-like (CML) proteins, calcineurin B-like proteins (CBL), and calcium-dependent protein kinases (CDPK) helps in $\mathrm{Ca}^{2+}$ signaling pathway during stress and non-stress condition. Deficiency symptoms occur more often in developing tissue such as young leaves and fruits, due to low remobilization from old to young tissue via the phloem. This leads to a strong dependency of $\mathrm{Ca}^{2+}$ supply via the xylem. Resulting physiological disorder are tip burn in lettuce or blossom end rot in tomato. Plants deficient in calcium have been shown to be more susceptible to pathogens and exogenous calcium supply in turn has been shown to improve the plant's resistance. Conserved microbial patterns for example PAMP initiating a signaling cascade leading to induction of downstream responses of defense gene expression. Future research will shed more light on the different functions of this important nutrient and how they are interconnected.

\section{Introduction}

Calcium is a chemical element with the symbol $\mathrm{Ca}$ and atomic number 20 . Ca is the $5^{\text {th }}$ most abundant element in the earth's crust after oxygen, silicon, aluminium and iron and the third most abundant metal, after iron and aluminium. Ca is under Group 2 in the periodic table. Calcium is a soft grey alkaline earth metal. Calcium is an essential macronutrient in plants, with concentrations in the shoot ranging from 0.1 to over $5 \%$ of 
dry wt (Marschner, 1995; White and Broadley, 2003). It is a leachable nutrient.

In plants, calcium is the third most abundant element after nitrogen and potassium. Calcium plays significant roles in plant growth and development as well as in adaptation to the diverse environments. Extracellular $\mathrm{Ca}^{2+}$ is critical in maintaining cell wall structure and function. Extracellular $\mathrm{Ca}^{2+}$ controls the stability and permeability of the plasma membrane through modulating its lipid compositions and retaining the functional integrity of the ion transporters. Plant plasma membranes have developed sophisticated sensing mechanisms to decode the dynamics of extracellular $\mathrm{Ca}^{2+}$. It is well documented that the cytoplasmic $\mathrm{Ca}^{2+}$ function as a second messenger mediating cellular responses to both external and internal clues. However, $\mathrm{Ca}^{2+}$ function is largely unknown in other subcellular organelles, such as mitochondria, Golgi, endoplasmic reticulum and nuclei. $\mathrm{Ca}^{2+}$ uptake and its re-distribution in cells depend on $\mathrm{Ca}^{2+}$ transporters that are distinct in binding affinity, flux direction, and energy utilization.

Once entering into plants, $\mathrm{Ca}^{2+}$ binds to an array of $\mathrm{Ca}^{2+}$-binding proteins which in turn activate the downstream signaling cascade. Given the fact that it is the $\mathrm{Ca}^{2+}$ redistribution and the transient rise in $\mathrm{Ca}^{2+}$ contents but not the absolute content that confers calcium function, deciphering the mysteries of calcium functions depends largely on in vivo $\mathrm{Ca}^{2+}$ assay techniques that are time and concentration sensitive. Moreover, calcium contents in vegetable foods contribute largely to both human and livestock calcium nutrition. Both genetic approaches and agronomic practices have been taken to increase biological availability of calcium in crops, fruits and vegetables.

\section{Forms of Calcium (Ca)}

\section{Forms taken up by plants}

Calcium is absorbed by plants as its $\mathrm{Ca}^{2+}$ ion. However, such absorption takes place from the soil solution and probably by root interception or contact exchange. Mostly, calcium can be readily transported to root surfaces by mass flow process, excepting in highly leached and un-limed soils. Calcium concentration in the soil solution is present about 10 times greater than that of $\mathrm{K}$ concentration. In-spite of presence of greater concentration of $\mathrm{Ca}$ in the soil solution, its uptake by plants is lower than that of $\mathrm{K}$ as the capacity of the uptake of $\mathrm{Ca}$ is limited to only by young root tips.

\section{Forms of Ca in soils}

Calcium is present in soils as its various forms viz. mineral particles, $\mathrm{CaCO}_{3}$, simple salts and exchangeable $\mathrm{Ca}$.

\section{$\mathrm{Ca}^{2+}$ uptake by plant}

Calcium is taken up from the soil solution through plasma membrane channels expressed in roots (White et al., 2002). However, most of these channels have been studied in the context of a potential role in signaling rather than nutrition. As an important nutrient, calcium has to be taken up and distributed within the plant; however, its function as messenger affects the way it can be taken from the root surface to the xylem, in which it is transported to the shoot. In principle, both the apoplastic and the symplastic pathway could be available for this movement, $\mathrm{Ca}^{2+}$ concentrations in the cytosol need to be in the submicromolar range to ensure the ability of the cell to generate a $\mathrm{Ca}^{2+}$ signal. Symplastic movement therefore would interfere with the signaling function of calcium. A common model suggests that $\mathrm{Ca}^{2+}$ 
moves apoplastically from the epidermis through the cortex until it reaches the casparian strip of the endodermis (White, 2001). The casparian strip forms a barrier around endodermal cells, which consists mainly of suberin and lignin and prevents movement of water and solutes in order to inhibit the uptake of unwanted or toxic substances (Schreiber et al., 1999). Thus, reaching this cell layer, $\mathrm{Ca}^{2+}$ will have to enter the cytosol of the endodermal cells via channel proteins and be exported into the stelar apoplast via $\mathrm{Ca}^{2+}$-ATPases or $\mathrm{Ca}^{2+} / \mathrm{H}^{+}$ antiporters to finally be loaded into the xylem. The identities of the channels mediating the influx into endodermal cells are currently unknown. In addition to this pathway, which involves the symplast to a certain extent, a purely apoplastic pathway has been proposed (White, 2001). This could occur in regions of the root where the casparian strip of the endodermis is not yet fully developed or interrupted, such as the root apex or at the sites of lateral root emergence. Interplay between the two pathways would saturate the need of both, nutrition of and signaling in root cells as well as transport of $\mathrm{Ca}^{2+}$ to the xylem for distribution to the shoot (White, 2001). Once loaded into the xylem, calcium is transported to the shoot, where it finally has to be unloaded into and distributed within the leaf cells (White and Broadley, 2003).

\section{Calcium transporter in cell membrane}

\section{$\mathrm{Ca}^{2+}$ eflux from the cytosol : $\mathrm{Ca}^{2+}-\mathrm{ATPase}$ and $\mathrm{H}^{+} / \mathrm{Ca}^{2+}$ antiporters}

The removal of $\mathrm{Ca}^{2+}$ from the cytosol against its electrochemical gradient to either the apoplast or to intracellular organelles requires energized, 'active' transport. This is catalysed by $\mathrm{Ca}^{2+}$-ATPases and $\mathrm{H}^{+} / \mathrm{Ca}^{2+}$-antiporters. By removing Calcium from cytoplasm these enzymes perform several important functions (Hirschi 2001):
(1) They maintain a low $\left[\mathrm{Ca}^{2+}\right]$ cyt in the resting (unstimulated) cell appropriate for cytoplasmic metabolism

(2) They restore $\left[\mathrm{Ca}^{2+}\right]$ cyt to resting levels following a $\left[\mathrm{Ca}^{2+}\right]$ cyt perturbation, thereby influencing the magnitude, kinetics and subcellular location of $\left[\mathrm{Ca}^{2+}\right]$ cyt signals

(3)They replenish intracellular and extracellular $\mathrm{Ca}^{2+}$ stores for subsequent $\left[\mathrm{Ca}^{2+}\right]$ cyt signals and permit the generation of local $\left[\mathrm{Ca}^{2+}\right]$ cyt oscillations through their interplay with $\mathrm{Ca}^{2+}$ channels

(4) They provide $\mathrm{Ca}^{2+}$ in the ER for the secretory system to function;

(5) They remove divalent cations, such as $\mathrm{Mg}^{2+}, \mathrm{Mn}^{2+}, \mathrm{Ni}^{2+}$ or $\mathrm{Zn}^{2+}$, from the cytosol,

to support the specialized biochemistry of particular organelles and to prevent mineral toxicities

\section{$\mathrm{Ca}^{2+}$ influx to the cytosol: Calcium channels}

Calcium-permeable channels have been found in all plant membranes. They have been classified on the basis of their voltagedependence into depolarization-activated (DACC), hyperpolarization-activated (HACC) and voltage independent (VICC) cation channels (Sanders et al., 2002). The presence of diverse classes of $\mathrm{Ca}^{2+}$-permeable channels in a particular membrane is thought to enable physiological flexibility. The principal roles of $\mathrm{Ca}^{2+}$-permeable channels in the plasma membrane appear to be in cell signalling, but they may also contribute to nutritional $\mathrm{Ca}^{2+}$ fluxes in particular cell types. Most DACCs activate significantly at voltages more positive than about \pm 150 to $\pm 100 \mathrm{mV}$ under physiological conditions. The outward-rectifying $\mathrm{K}^{+}$channels (KORCs) found in the plasma membrane of plant cells are also $\mathrm{Ca}^{2+}$-permeable DACCs. These channels activate significantly at voltages more positive than about $\pm 50 \mathrm{mV}$ under most physiological conditions and catalyse a large 
$\mathrm{K}^{+}$eflux simultaneously with a small $\mathrm{Ca}^{2+}$ influx. Many distinct VICCs are present in the plasma membrane of plant cells, which differ in cation selectivity, voltage dependence and pharmacology (White et al., 2002). Nevertheless, all VICCs appear to be permeable to both monovalent and divalent cations and are likely to provide a weakly voltage-dependent $\mathrm{Ca}^{2+}$ influx to cells under physiological ionic conditions. It has been suggested that $\mathrm{Ca}^{2+}$ influx through VICCs, which are open at physiological voltages and are generally insensitive to cytoplasmic modulators, is required to balance the perpetual $\mathrm{Ca}^{2+}$ efflux through $\mathrm{Ca}^{2+}$-ATPases and $\mathrm{H}^{+} / \mathrm{Ca}^{2+}$-antiporters to maintain $\left[\mathrm{Ca}^{2+}\right]$ cyt homeostasis in an unstimulated plant cell.

\section{Physiological roles of calcium}

\section{Cell wall formation}

In cell wall the region where Calcium is essential is Middle lamella. The middle lamella is a layer which cements the cell walls of two adjoining plant cells together. The middle lamella is made up of calcium pectates. In a mature plant cell it is the outermost layer of cell wall.

\section{Maintain stability of cell membrane}

$\mathrm{Ca}^{2+}$ by binding to phospholipids, stabilizes the lipid bilayers and thus provides structural integrity to cellular membrane.

\section{Calcium as second messenger in signaling network}

Cell signaling is a fundamental process by which specific information is transferred from cell surface to the cytosol and ultimately to the nucleus. Second messengers are molecules that relay signals from receptors on the cell surface to target molecules inside the cell. While early work concentrated on the role of calcium as a nutrient and structural component, it has become more and more clear now that the main function of calcium lies in its ability to serve as a second messenger in a vast variety of physiological, developmental, and stress-related processes, and indeed some of the observed effects of calcium application might also be connected to this function rather than being purely structural. The generation of a Calcium signal is the result of a complex interplay between influx channels and exporters as well as pumps. As calcium signals are involved in a diverse range of processes, it is assumed that the spatiotemporal pattern of the calcium rise, the so-called Calcium signature, determines the specificity of the corresponding response (McAinsh and Pittman, 2009; Dodd et al., 2010). Different sets of proteins may determine the signature in response to different cues, although some components might be involved in several responses. As important as the players generating the signal are those that decode it into (specific) downstream responses. These are calmodulin (CaM), CaM-like proteins (CMLs), calcineurin B-like proteins (CBLs), CBL interacting protein kinases (CIPKs) as well as $\mathrm{Ca}^{2+}$-dependent kinases (CDPKs or CPKs in Arabidopsis). While CaM, CMLs, CBLs, and CDPKs contain EF-hand $\mathrm{Ca}^{2+}$-binding motifs, CIPKs are activated by the interaction with CBLs (Kudla et al., 2018). Activation of downstream targets such as transcription factors, transporters, or channels then further relays the signal into outputs such as gene expression or stomatal closure.

\section{Calcium as second messenger in signaling network}

Cell signaling is a fundamental process by which specific information is transferred from cell surface to the cytosol and ultimately to the nucleus. Second messengers are molecules that relay signals from receptors on 
the cell surface to target molecules inside the cell. While early work concentrated on the role of calcium as a nutrient and structural component, it has become more and more clear now that the main function of calcium lies in its ability to serve as a second messenger in a vast variety of physiological, developmental, and stress-related processes, an

\section{Calcium as second messenger in signaling network}

Cell signaling is a fundamental process by which specific information is transferred from indeed some of the observed effects of calcium application might also be connected to this function rather than being purely structural. The generation of a Calcium signal is the result of a complex interplay between influx channels and exporters as well as pumps. As calcium signals are involved in a diverse range of processes, it is assumed that the spatiotemporal pattern of the calcium rise, the so-called Calcium signature, determines the specificity of the corresponding response (McAinsh and Pittman, 2009; Dodd et al., 2010). Different sets of proteins may determine the signature in response to different cues, although some components might be involved in several responses. As important as the players generating the signal are those that decode it into (specific) downstream responses. These are calmodulin (CaM), CaM-like proteins (CMLs), calcineurin B-like proteins (CBLs), CBL interacting protein kinases (CIPKs) as well as $\mathrm{Ca}^{2+}$-dependent kinases (CDPKs or CPKs in Arabidopsis). While CaM, CMLs, CBLs, and CDPKs contain EF-hand $\mathrm{Ca}^{2+}$-binding motifs, CIPKs are activated by the interaction with CBLs (Kudla et al., 2018). Activation of downstream targets such as transcription factors, transporters, or channels then further relays the signal into outputs such as gene expression or stomatal closure.

\section{$\mathrm{Ca}^{2+}$ is involved in the processes of photosynthetic reaction}

\section{Helps in Photolysis of water}

Photosystem 2 (PS2) is composed of membrane-related redox enzymes, and $\mathrm{Ca}^{2+}$ acts as a cofactor to participate in the formation of activation sites. Oxygenevolving complex (OEC), a component of PS2, is involved in the decomposition of water molecules (Dau et al., 2007). Extrinsic PsbQ, PsbP, and PsbO are OEC proteins. PsbQ and PsbP require $\mathrm{Cl}^{-}$and $\mathrm{Ca}^{2+}$ as essential co-factors (Pagliano et al., 2009). PsbO-associated $\mathrm{Ca}^{2+}$ is from the $\mathrm{Mn}_{4} \mathrm{CaO}_{5}$ cluster, and PsbO is closely related to the stability of the $\mathrm{Mn}_{4} \mathrm{CaO}_{5}$ cluster. $\mathrm{Ca}^{2+}$ also participates in the s-state cycle, which is associated with water decomposition. Many studies have suggested that the $\mathrm{Mn}_{4} \mathrm{CaO}_{5}$ cluster is a necessary precondition for water oxidation. OEC splits water into oxygen molecules, protons, and electrons. Subsequently, electrons are transported to $\mathrm{NADP}^{+}$, generating NADPH via linear electron flow (LEF); LEF probably generates ATP and NADPH for the Calvin cycle. In this process, photosystem 1 (PS1) can transfer electrons of ferredoxin (FD) to $\mathrm{NADP}^{+}$and form NADPH through ferredoxin $\mathrm{NADP}_{+}$ oxidoreductase (FNR). However, if FD does not transfer electrons to NADP+ but passes through plastoquinone (PQ) to PS1 again, then this mode of electron transfer is called cyclic electron flow (CEF). PS1 participates in both LEF and CEF electron transfer, which plays an important role in the formation of CEF in electron transfer. PS1 is composed of multiple subunits (e.g., PsaA, -N, and -H). PsaN regulates photosynthetic electron flow through $\mathrm{Ca}^{2+}$-dependent phosphorylation (Reddy et al., 2002), and it may also be related to electron transport from plastocyanin (PC) to PS1. PsaL and PsaA may also be associated with calcium. FD is involved in the 
electron transport of PS1 and has high affinity with $\mathrm{Ca}^{2+}$ in its reduced state. In addition, FD can interact with PsaD, PsaE, PsaC, and PsaH.

\section{Calcium as second messenger in signaling network}

Cell signaling is a fundamental process by which specific information is transferred from Regulating Photosynthetic Enzyme Activity of Carbon Assimilation.The Calvin cycle is the main pathway of carbon assimilation, and it occurs on the stroma of the chloroplast. Sedoheptulose-1,7-bisphosphatase (SBPase) and fructose-1,6-bisphosphatase (FBPase) are the two key enzymes in the Calvin cycle (Raines, C.A. 2003), and their activities are regulated by $\mathrm{Ca}^{2+}$. The two types of FBPase are cytoplasm FBPase and chloroplast FBPase (Rojas-González et al., 2015). The decrease in activity of chloroplast FBPase and SBPase can reduce the chloroplast content and inhibit plant growth, and the absence of these two enzymes in higher plants may damage photosynthesis (Steal et al., 2007). Even though $\mathrm{Ca}^{2+}$ can regulate carbon assimilation by mediating these two enzymes, high concentrations of exogenous $\mathrm{Ca}^{2+}$ can inhibit carbon assimilation. Transketolase (TKL) is another key enzyme of the Calvin cycle, which occurs in the chloroplast. It is involved in the regeneration of various substances in the Calvin cycle, such as erythrose4phosphate (E4P) and xylulose5-phosphate (X5P). TKL was found to be phosphorylated in the chloroplast extract and was speculated to be related to the $\mathrm{Ca}^{2+}$-dependent pathway (Rocha et al., 2014)

\section{$\mathrm{Ca}^{2+}$ is involved in the mechanisms of regulating photoprotection}

The photosynthetic proteins are damaged under light stress in an event called photodamage, such as the net loss of D1 protein. Photoprotection refers to the capacity of preventing damage to the photosystem under excess energy, including the consumption of excess light energy and the removal of reactive oxygen species. During photosynthesis, the captured light energy is mainly used by photochemical electron transfer, chlorophyll fluorescence emission, and heat dissipation. Photochemistry electron transfer is associated with the synthesis of photosynthetic products, and chlorophyll fluorescence emission is only rarely part of light energy consumption. Thus, heat dissipation is an important way to consume excess light energy and prevent photodamage. The process of dissipating harmlessly excess excitation energy as heat is called NPQ. Some reports showed NPQ is regulated by $\mathrm{Ca}^{2+}$ (Bialasek et al., 2017).

The recombination of the PS2 reaction center complex is a common possible mechanism of NPQ, which involves reversible inactivation of D1 protein and synthetic regeneration. High contents of D1 protein have been detected in $\mathrm{Ca}^{2+}$-treated plants (Yang et al., 2013), and the turnover of protein components of photosynthetic reaction centers is regulated by $\mathrm{CaM}$, an important component of $\mathrm{Ca}^{2+}$ signal transduction pathway. Moreover, $\mathrm{Ca}^{2+}$-binding sites may exist on D1 protein. PsbS, a nuclear encoded PS2 subunit protein, also plays a key role in NPQ, because $\mathrm{Ca}^{2+}$ can induce the aggregation of $\mathrm{PsbS}$ in vitro.

The ROS scavenging system can help plant cells maintain the balance of the ROS content. ROS can inhibit D1 protein recombination (Nishiyama et al., 2014). Exogenous $\mathrm{Ca}^{2+}$ can activate ascorbate peroxidase (APX), catalase (CAT), and superoxide dismutase (SOD) during heat stress (Yang et al., 2015). Thus, the $\mathrm{Ca}^{2+}$ signal transduction pathway is involved in regulating ROS balance and protecting the photosystem. 
Mechanisms of $\mathrm{Ca}^{2+}$ involved in stomatal regulation

Stomata are important channels for plants to communicate with the environment, especially during photosynthesis. Each stoma comprises a pair of guard cells with a small amount of chloroplasts, and these chloroplasts are related to stomatal movement. As a key signal of stomatal regulation, ROS is mainly produced in chloroplasts (Song et al., 2014). The production of hydrogen peroxide $\left(\mathrm{H}_{2} \mathrm{O}_{2}\right)$ induced by abscisic acid (ABA) in guard cell chloroplasts is earlier than that in other locations. $\mathrm{Ca}^{2+}$ sensor (CAS), which is located on the thylakoid membranes of a chloroplast, is a $\mathrm{Ca}^{2+}$-binding protein (Petroutsos et al., 2011) associated with the production of $\mathrm{H}_{2} \mathrm{O}_{2}$ and nitric oxide (NO) in the stomatal closure pathway. $\mathrm{H}_{2} \mathrm{O}_{2}$ affects stomatal movement by activating $\left[\mathrm{Ca}^{2+}\right]$ cyt channels, and CAS is involved in the generation of $\mathrm{H}_{2} \mathrm{O}_{2}$, which induces $\left[\mathrm{Ca}^{2+}\right]$ cyt oscillation by activating $\left[\mathrm{Ca}^{2+}\right]$ cyt channels and then causes stomatal closure Some reports showed that $\left[\mathrm{Ca}^{2+}\right] \mathrm{cyt}$ oscillations can also be induced by the inositol 1,4,5-trisphosphate (IP3) under external stress (Wang et al., 2008) and this phenomenon is related to CAS. Additionally, NADPH oxidases RbohD and RbohF, which are also known as respiratory burst oxidase homologues, are both involved in stomatal movement. Moreover, they have two $\mathrm{Ca}^{2+}$ binding EF-hand motifs. RbohD/F and phosphatidic acid (PA) produced by phospholipase D (PLD) are related to ROS production, and the PLDa1-null Arabidopsis mutant and rbohD/F depletion mutant are insensitive to the pathway in which $\mathrm{ABA}$ induces stomatal closure. RbohD/F and PA are speculated to function in the same pathway. Further research showed that PA interacts with $\mathrm{RbohD} / \mathrm{F}$ to increase NADPH activity, thereby affecting ROS production. In addition, NO is involved in this pathway and plays a vital role. When the activities of phospholipase C (PLC) and PLD are inhibited, NO-induced stomatal closure is also prevented. Thus, NO acts upstream in the PLC and PLD pathway. However, different studies showed that NO occurs in the downstream events of the pathway, in which PLD generates PA. Stomatal movement is coregulated by a signal network including the $\mathrm{Ca}^{2+}$ signal transduction pathway, plant hormone pathway, and ROS signal pathway, but the relationship between NO and PA on the stomatal movement pathway needs further research.

In conclusion, calcium is an essential element in plants. $\mathrm{Ca}^{2+}$ contributes to the structure of cells, photosynthetic reaction, photoprotection (D1 protein, PsbS Regulated by $\mathrm{Ca}^{2+}$ ) and also helps in stomatal movement. It works as secondary messenger molecules that relay signals from receptors on the cell surface to target molecules inside the cell. Signaling events connected to a vast variety of physiological, developmental changes in plants. CaM, CMLs and CDPKs complexes form intricate signaling networks for translating these signatures into phosphorylation events and transcriptional responses.

\section{References}

Białasek, M., Górecka, M., Mittler, R., Karpi' nski, S.(2017). Evidence for the involvement of electrical, calcium and ros signaling in the systemic regulation of non-photochemical quenching and photosynthesis. Plant Cell Physiol. 58, 207-215

Dau, H., Haumann, M. (2007). Eight steps preceding $\mathrm{O}-\mathrm{O}$ bond formation in oxygenic photosynthesis-a basic reaction cycle of the photosystem ii manganese complex. Biochim. Biophys. Acta. , 1767, 472.

Dodd, A. N., Kudla, J., and Sanders, D. 
(2010). The language of calcium signaling. Annu. Rev. Plant Biol. 61, 593-620. doi: 10.1146/annurev-arplant070109-104628

Hirschi K. (2001). Vacuolar $\mathrm{H}+/ \mathrm{Ca}^{2+}$ transport: Who's directing the traffic? Trends in Plant Science 6: 100-104.

Kudla, J., Becker, D., Grill, E., Hedrich, R., Hippler, M., Kummer, U., et al., (2018). Advances and current challenges in calcium signaling. New Phytol. 218, 414-431. doi: 10.1111/nph.14966

Marschner, H. (1995). Mineral nutrition of higher plants. (San Diego: Academic Press).

McAinsh, M. R., and Pittman, J. K. (2009). Shaping the calcium signature. New Phytol. 181, 275-294. doi: 10.1111/j.1469-8137.2008.02682.

Nishiyama, Y., Yamamoto, H., Allakhverdiev, S.I., Inaba, M., Yokota, A., Murata, N. (2014). Oxidative stress inhibits the repair of photodamage to the photosynthetic machinery. EMBO J. 20, 5587-5594.

Pagliano, C., Rocca, N.L., Andreucci, F., Deák, Z., Vass, I., Rascio, N., Barbato, R. (2009). The extreme halophyte Salicornia veneta is depleted of the extrinsic PsbQ and PsbP proteins of the oxygen-evolving complex without loss of functional activity. Ann. Bot. 103, $505-515$.

Petroutsos, D., Busch, A., Janssen, I., Trompelt, K., Bergner, S.V., Weinl, S., Holtkamp, M., Karst, U., Kudla, J., Hippler, M. (2011). The chloroplast calcium sensor CAS is required for photoaccmilaation in Chlamydomonas reinhardtii. Plant Cell, 23, 2950.

Raines, C.A. (2003). The Calvin cycle revisited. Photosynth. Res. 75, 1-10.

Reddy, V.S., Ali, G.S., Reddy, A.S.(2002) Genes encoding calmodulin-binding proteins in the Arabidopsis genome. J.Biol. Chem. 277, 9840-9852.
Rocha, A.G., Mehlmer, N., Stael, S., Mair, A., Parvin, N., Chigri, F., Teige, M., Vothknecht, U.C.(2014) Phosphorylation of Arabidopsis transketolase at Ser428 provides a potential paradigm for the metabolic control of chloroplast carbon metabolism. Biochem. J. 458, 313.

Rojas-González, J.A., Soto-Súarez, M., García-Díaz, Á., Romero-Puertas, M.C., Sandalio, L.M., Mérida, Á., Thormählen, I., Geigenberger, P., Serrato, A.J., Sahrawy, M.(2015) Disruption of both chloroplastic and cytosolic FBPase genes results in a dwarf phenotype and important starch and metabolite changes in Arabidopsis thaliana. J. Exp. Bot. 66, 2673-2689.

Sanders D, Pelloux J, Brownlee C, Harper JF (2002). Calcium at the crossroads of signaling. Plant Cell 14: S401-S417

Schreiber, L., Hartmann, K., Skrabs, M., and Zeier, J. (1999). Apoplastic barriers in roots: chemical composition of endodermal and hypodermal cell walls. J. Exp. Bot. 50, 1267-1280.

Song, Y., Miao, Y., Song, C.P.(2014). Behind the scenes: the roles of reactive oxygen species in guard cells. New Phytol. 201, 1121-1140.

White, P. J., Bowen, H. C., Demidchik, V., Nichols, C., and Davies, J. M. (2002). Genes for calcium-permeable channels in the plasma membrane of plant root cells. Biochim. Biophys. Acta Biomembr. 1564, 299-309. doi: 10.1016/ S0005-2736(02)00509-6

White, P. J. (2001). The pathways of calcium movement to the xylem. J. Exp. Bot. 52, 891-899. doi: 10.1093/jexbot/ 52.358 .891

White, P. J., and Broadley, M. R. (2003). Calcium in plants. Ann. Bot. 92, 487511. doi: 10.1093/aob/mcg164

Wang, C.R., Yang, A.F., Yue, G.D., Gao, Q., Yin, H.Y., Zhang, J.R. (2008). 
Enhanced expression of phospholipase C 1 (ZmPLC1) improves drought tolerance in transgenic maize. Planta 227, 1127.

Yang, S., Wang, F., Guo, F., Meng, J.J., Li, X.G., Dong, S.T., Wan, S.B. (2013). Exogenous calcium alleviates photoinhibition of PSII by improving the xanthophyll cycle in peanut
(Arachis hypogaea) leaves during heat stress under high irradiance. PLOS ONE, $8, \mathrm{e} 71214$.

Yang, S., Wang, F., Guo, F., Meng, J.J., Li, X.G., Wan, S.B. (2015). Calcium contributes to photoprotection and repair of photosystem II in peanut leaves during heat and high irradiance. J. Integr. Plant Biol. 57, 486-495.

\section{How to cite this article:}

Mainu Hazarika1 and Praveen Kumar. 2020. Physiology of Calcium Nutrition in Plants. Int.J.Curr.Microbiol.App.Sci. 9(10): 841-849. doi: https://doi.org/10.20546/ijcmas.2020.910.100 